\title{
Fig (Ficus Carica L.) Identification Based on Mutual Information and Neural Networks
}

\author{
Ghada Kattmah \\ General Commission of Agricultural Scientific Research, Department of Hort iculture science, Syria \\ e-mail: Ghada978@Gmail.com \\ Gamil Abdel Azim \\ College of Computers \& Informatics, Canal Suez University, Egypt \\ e-mail: gazim3@gmail.com
}

\begin{abstract}
The process of recognition and identification of plant species is very time-consuming as it has been mainly carried out by botanists. The focus of computerized living plant's identification is on stable feature's extraction of plants. Leaf-based features are preferred over fru its, also the long period of its existence than fruits. In this preliminary study, we study and propose neural networks and Mutual information for identification of two, three Fig cultivars (Ficus Carica L.) in Syria region. The identification depends on image features of Fig tree leaves. A feature extractor is designed based on Mutual Information computation. The Neural Networks is used with two hidden layers and one output layer with 3 nodes that correspond to varieties (classes) of FIG leaves. The proposal technique is a tester on a database of 84 images leaves with 28 images for each variety (class). The result shows that our technique is promising, where the recognition rates $100 \%$, and $92 \%$ for the training and testing respectively for the two cultivars with $100 \%$ and 90 for the three cultivars. The preliminary results obtained indicated the technical feasibility of the proposed method, which will be applied for more than 80 varieties existent in Syria.
\end{abstract}

Index Terms - Pattern recognition, Texture analysis, neural network, mutual information, Fig tree classification and identification

\section{INT RODUCTION}

The computerization of plant management species becomes more popular. The process of recognition and identification of plant species is very time-consuming as it has been mainly carried out by botanists. The focus of computerized living plant identification is on stable feature's extraction of plants. The information of leaf plays an important role in identifying living plants. Leaf-based features are preferred over owner's fruits, etc. due to the seasonal nature of the later and also the abundance of leaves. Fig (Ficus Carica L.) tree plays an important role in the Syrian economy, where Syria produces 42944 tons a year. For this reason, the classification of fig tree species is of paramount importance to preserve type and improve production. In this research, we described the classification of a plant Fig (Ficus Carica L) with multiple varieties. Fig is one of the oldest fruit trees in the Mediterranean zone. Its wild genetic resource still exists in many countries, including Syria and Anatolia, which are considered as the natural habitats of the fig tree.

The classification of plant leaf has been introduced in several approaches such as k-Nearest Neighbor Classifier (k-NN), Probabilistic Neural Net work (PNN), Genetic Algorithm (GA), Support Vector Machine (SVM), and Principal Component Analysis (PCA) [1]. Zulkifli [2] proposed General Regression Neural Network to classify 10 kinds of plants with green color leaves. Wu et al. [3] used PNN to classify 32 kinds of green leaves. Singh et al. [4] suggested SVM to implement a classifier for the problem. Shabanzade et al. [5] used Linear Discriminant Analysis (LDA). Several of researchers are used aspect ratio, leaf vein, and invariant mo ment to identify plant. Several features such as an aspect ratio (ratio between length and width of leaf), the ratio of perimeter to the diameter of leaf, and vein features were used to characterize the leaf with accuracy of $90 \%$. Wu et al. [3, 6]

On the other hand, Texture analysis is important in many applications of computer image analysis for classification, detection, or segmentation of images based on local spatial variations of the intensity or the color. Actually, shape, color and texture features are common features involved in several applications, such as in [7] and [8]. However, some researchers used part of those features only. Invariant moments proposed by Hue are very popular in image processing to recognize objects, including leaves of plants [9-10]. Polar Fourier transforms (PFT) proposed by Zhang [2]. Several leaf classification systems have incorporated texture features to improve the performance, such as in [12] that used entropy; homogeneity and contraction derived from the co-occurrence matrix came from Digital Wavelet Transform (DWT), in [13] that used lacunarity to capture the texture of leaf and in [14] that used GLCM.

Texture features can be extracted by using various methods. Gray-level Gabor Filter and Local binary 
pattern (LBP) are examples of popular methods to extract texture features.

To implement the preliminary classification system, in this research, we tried to capture the texture of the leaf by a new feature extraction technique based on mutual information. Those features were inputted into the identification system that uses a neural network classifier. Testing was done by using a data set. The result shows that method improves good performance (92\%) of the identification system with 2 cultivars and 90 with 3 cultivars.

Mutual Information (MI) and Entropy provide an intuitive tool to measure the uncertainty of random variables and the information shared by them, in which the entropy and the mutual information are two critical concepts. The entropy is a measure of the uncertainty of random variables, while the mutual information is a measure of information shared by two random variables. MI can compute between two variables if we have explicit knowledge of the probability distributions. In general, these probabilities are not known. Various methods are used to estimate the probability densities from the observed data. In the next two sections, this paper treats a brief presentation of MI concept and the descriptor algorithm. In the section 4, the concept of neural networks Training Multilayer Perceptrons are described. Image analysis is given in sections 5. Results and discussion are presented in section 6. At the end, a conclusion is presented.

\section{ENTROPY AND MUTUAL INFORMATION}

The entropy $\mathrm{H}$ is a measure of the uncertainty of random variables. Let $X$ is a discrete random variable with alphabet and $p(x)=P(X=x), x \in X$ be the probability mass function, the entropy of $\mathrm{X}$ is defined as:

$$
H(\mathrm{X})=-\sum_{x \in \mathrm{X}} p(x) \log p(x)
$$

While the mutual information is a measure of information shared by two random variables, defined as:

$$
\begin{aligned}
I(X, Y) & =\sum_{x \in X} \sum_{y \in Y} p(x, y) \log \frac{p(x, y)}{p(x) p(y)} \\
& =H(Y)-H(Y \mid X),
\end{aligned}
$$

Where $p(x, y)$ represents the joint probability distribution of $(\mathrm{X}, \mathrm{Y}), \quad p(x)$ and $p(y)$ are the marginal distributions of $X$ and $Y$, respectively. $H(Y \mid X)$ is the conditional entropy of $\mathrm{Y}$ in the case of $\mathrm{X}$ is known. It can be represented as:

$$
H(Y \mid X)=-\sum_{x \in X} p(x, y) \log p(y \mid x)
$$

For the continuous random variables, the entropy and the MI are defined as in (1), (2) and (3) after replaced the summation by integration. The MI is zero if and only if $\mathrm{X}$ and $\mathrm{Y}$ are statistically independent, i.e. vanishing mutual information does imply that the two variables are independent. This shows that MI provides a more general measure of dependencies in the data, in particular, positive, negative and nonlinear correlations.

We can compute the MI between two variables if we have explicit knowledge of the probability distributions. In general, these probabilities are not known. Various methods are used to estimate the probability densities from the observed data. Consider two sequences $\left(\mathrm{S}_{\mathrm{i}}\right)$ and $\left(\mathrm{S}_{\mathrm{J}}\right)$ of $\mathrm{n}$ simultaneous observations of two random variables. Since entropy is computed using discrete probabilities, we estimate probability densities using the widely used histogram method [16-20].

Let $\mathrm{Fx}(\mathrm{i})$ denote the number of observations of $\mathrm{X}$ falling in the bin $\mathrm{a}_{\mathrm{i}}$. The probabilities $\mathrm{p}\left(a_{i}\right)$ are then estimated as:

$$
\mathrm{p}\left(a_{i}\right)=\frac{f_{X}(i)}{n}
$$

Let $\mathrm{f}_{\mathrm{Y}}(j)$ denote the number of observations of $\mathrm{Y}$ falling in the bin $b_{j}$. The probabilities $\mathrm{p}\left(b_{j}\right)$ are then estimated as:

$$
\mathrm{p}\left(b_{j}\right)=\frac{f_{Y}(j)}{n}
$$

Let $f_{X Y}(i ; j)$ denote the number of observations such as that $\mathrm{X}$ falls in bin $\boldsymbol{a}_{\boldsymbol{i}}$ and $\mathrm{Y}$ falls in bin $\boldsymbol{b}_{\boldsymbol{j}}$. Then the mutual information between $\mathrm{X}$ and $\mathrm{Y}$ is estimated as:

$$
I(X, Y)=\log n+\frac{1}{n} \sum_{i} \sum_{j} f_{X Y}(i ; j) \log \frac{f_{X Y}(i ; j)}{f_{X}(i) f_{Y}(j)}
$$

\section{NEW DESCRIPTOR BASED ON MUTUAL INFORMATION.}

In the following section, we describe our new Algorithm NDBMI for constructing the descriptor.

1- Given a image (matrix) $\mathrm{A}(\mathrm{n}, \mathrm{m})$, divide it in to sub images (matrices) $S_{i}(k, l)$, it gives a collection of sub images (matrices) $\mathrm{P}$

2- Find $\rho$ the mean matrix of the collection $\mathrm{P}$

3- Calculate the MI between each sub matrix in the collection $\mathrm{P}$ and the mean matrix; it gives a vector with dimension equal to cardinality of the collection. 
4- Consider the vector calculated in 3 as a descriptor of the image A.

\section{NEURAL NETWORKS}

In the following sub section we present and review one of the important classes of ANN, which is the Feed Forward Neural Networks.

Artificial Neural Networks (ANNs) are massively parallel, highly connected structures consisting of a number of simple, non linear processing units. Due to their massively parallel structure, they can perform computation at very high rate if implemented on a dedicated hardware. Moreover of their adaptive nature, they can learn the characteristics of inputs signals and adapt to data changes. The nonlinear nature of A NNs can help in performing function approximation and signal filtering operations which are beyond optimal linear techniques.

\section{A. feed-forward artificial neural networks}

Artificial neural networks are parallel computational models which are able, at least in principle, to map any nonlinear functional relationship between an input and an output hyperspace to the desired accuracy. They are constituted by individual processing units called neurons or nodes and differ among each other in the way these units are connected to process the information (architecture) and, consequently, in the kind of learning protocol adopted. In particular, the neurons of a feedforward neural network are organized in three layers: The input units receive information from the outside world, usually in the form of a data file; the intermediate neurons, contained in one or more hidden layers, allow nonlinearity in the data processing; the output layer is used to provide an answer for a given set of input values (Fig. 1).

In a fully connected artificial neural network, each neuron in a given layer is connected to each neuron in the following layer by an associated numerical weight Wij. The weight connecting two neurons regulates the magnitude of signal that passes between them. In addition, each neuron possesses a numerical bias term, corresponding to an input- $\boldsymbol{\phi} \mathrm{f}$ whose associated weight has the meaning of a threshold value. Information in an ANN is stored in these connection weights which can be thought of as the "memory" of the system. The goal of back-propagation training is to change iteratively the weights between the neurons in a direction that minimizes the error $E$, defined as the squared difference between the desired and the actual outcomes of the output nodes, summed over the training patterns (training set data) and the output neurons, according to the steepest descent method:

$$
\Delta W_{i j}(k)=-\eta \frac{\partial E}{\partial w_{i j}}+\alpha \Delta W_{i j}(k-1)
$$

The variation of a connection weight at the $\mathrm{k}^{\text {th }}$ iteration depends on the partial derivative of the total error with respect to that weight through a proportionality constant termed learning rate $(\eta)$ and on the variation of the same weight during the previous iteration by means of a momentum term $(\alpha)$. To compute the partial derivative of the error $E$ with respect to the connection weights to the hidden layer(s) requires propagating backwards the prediction error E using the rules of chain derivation, hence, the name "backpropagation": Chain derivation acts as a way to "distribute" the error E between the neurons of the hidden layer(s) in order to apply the iterative weight adjustment, necessary for the learning of the network [21-23].

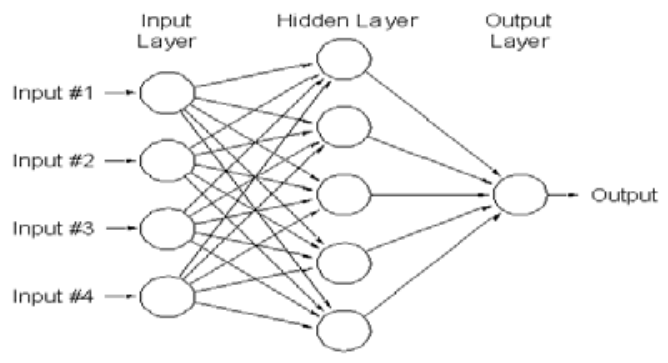

Figure 1: Feed-forward Neural with three layers

\section{B. Training Multilayer Perceptrons}

Once the number of layers, and number of units in each layer, has been selected, the network's weights and thresholds must be set so as to minimize the prediction error made by the network. This is the role of the training algorithms. Which are used to adjust automatically the weights and thresholds in order to minimize this error. This process is equivalent to fitting the model represented by the network to the training data available. The error of a particular configuration of the network can be determined by running all the training cases through the network, comparing the actual output generated with the desired or target outputs. The differences are combined together to form an error function which measures the network error. One of the error functions is the sum squared error. [24-28]

\section{IMAGE ANALYSIS (FEATURE EXTRACTION \& PREPROCESSING DATA).}

We are interesting to apply the Mutual Information computation to identify and classify the Figs (Ficus Carical) trees in Syria region. Each pattern (Fig leaf image) is described using varieties of numeric features, which is the features vector returned by the feature extraction function based on mutual information descriptor (section 3). The data set is divided into two sets, one for training, and one for testing. The preprocessing parameters were determined using the matrix containing all features which used for training or testing; these same parameters were used to preprocess the test feature vectors before passing them to the trained neural network. 
A fixed number (m) of instances for each class were randomly assigned to the training set and the others instances from each class for testing set. The first preprocessing function was used to preprocess the networks training set, By normalizing the inputs and targets, they have means of zero and standard deviation of 1.The normalized training sets were used to train a Feed Forward Back Propagation network (with a number of inputs equal to the number of features (m), $(2 m+1)$ hidden neurons and 2 outputs neurons equal to the number of classes).

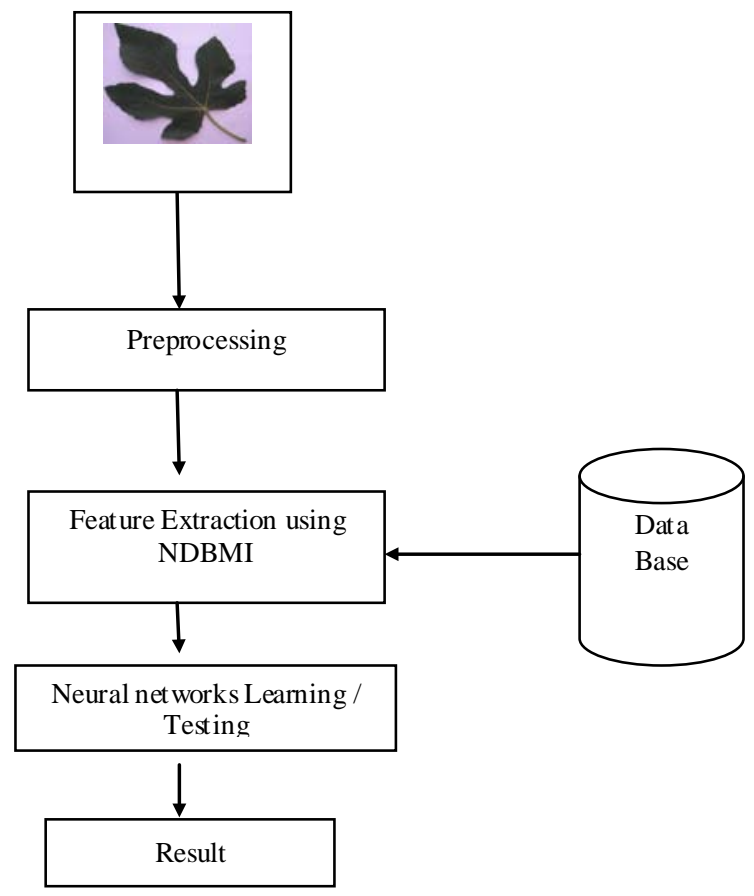

Figure.2 Model of the Leaf Classification System

\section{RESULTS AND DISCUSSION}

Fourty Two adult leaves of FIG were harvested from three trees in the same day all the leaves were chosen by experts in the same way, from those at a mature stage, showing no damage. The image of inferior leaf sides was only used, that is low resolution digital image. The momentum and learning rate were 0.9 and 0.01 respectively ( $\eta, \alpha$ in the equation 1$)$.

The output of the Neural Network is represented by a vector that belongs to $[0,1] n$, where $n$ is equal to the number of neurons in the output layer. It is also equal to the number of classes (2 or 3 ). For $n=2$, if the output vector is $(0,1)^{\mathrm{T}}$ then the networks classifies the pattern to the class two. The output vector should contain a one and only a one, Otherwise to the class one. We represent all features for the learning set in a matrix FM. Each column represents the pattern feature. If the learning set contains $d$ instances for each pattern that belongs to the same class, the dimension of the matrix FM will be equal to $m \times d \times n$ where $m$ is the number of features (dimension of feature vector) and $n$ is the number of classes. The columns number $1,2,3, \ldots, \mathrm{d}$ in the matrix
FM represents the instance features of the pattern (Fig leafs) that belongs to the class 1 . The columns number $d+1, d+2, d+3, \ldots, 2 d$, represents the instance features of the pattern (Fig leaf) which belongs to the class 2, etc. we rewrite the matrix FM as the form :

$$
\begin{aligned}
& F M={ }^{l} f_{i j} \text { for } l=1, .2,3, . ., d \\
& i=1,2,3, . ., m, \text { and } j=1,2,3, . ., n
\end{aligned}
$$

For example when $\mathrm{m}=6, \mathrm{~d}=2$ and $\mathrm{n}=4$, the outputs matrix OM will be as the fowling form (The neurons number of the input layer is equal 6 , and the output layer is equal 4)

$$
O M=\begin{array}{llllllll}
1 & 1 & 0 & 0 & 0 & 0 & 0 & 0 \\
0 & 0 & 1 & 1 & 0 & 0 & 0 & 0 \\
0 & 0 & 0 & 0 & 1 & 1 & 0 & 0 \\
0 & 0 & 0 & 0 & 0 & 0 & 1 & 1
\end{array}
$$

Also we represent all features for the testing set in a matrix TM each column rep resents the pattern feature. If the testing set contains dT instances for each pattern that belongs to the same class, The dimension of the matrix TM will be equal to $m \times d T \times n$ where $\mathrm{m}$ is the number of features (dimension of feature vector) and $n$ is the number of classes we rewrite the matrix TM as the form [29].

$$
\begin{aligned}
& T M={ }^{l} f_{i j} \quad \text { for } l=1, .2,3, . . . . d T \\
& i=1,2,3, \ldots ., m, \text { and } j=1,2,3, \ldots, n
\end{aligned}
$$

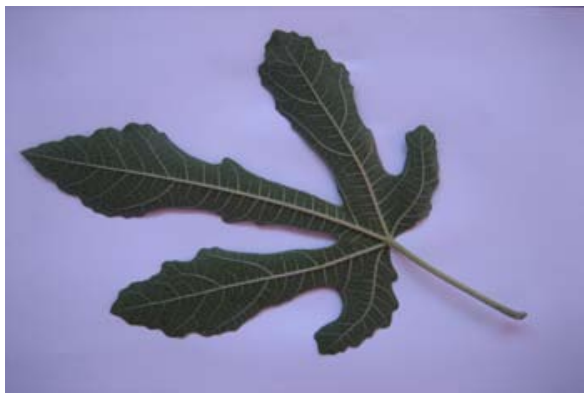

Figure.3 Example of image Fig tree class 1 (shaghoury)

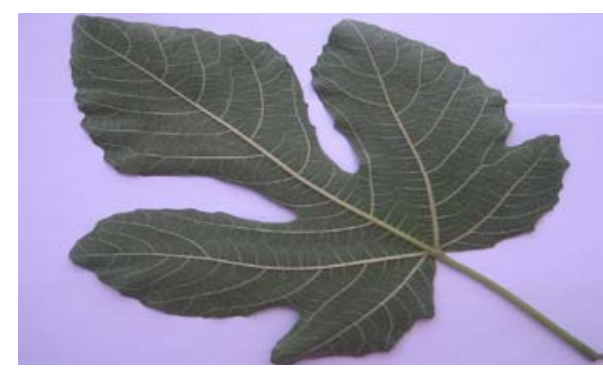

Figure.4 Example of image Fig tree class 2 (Dir Attia) 


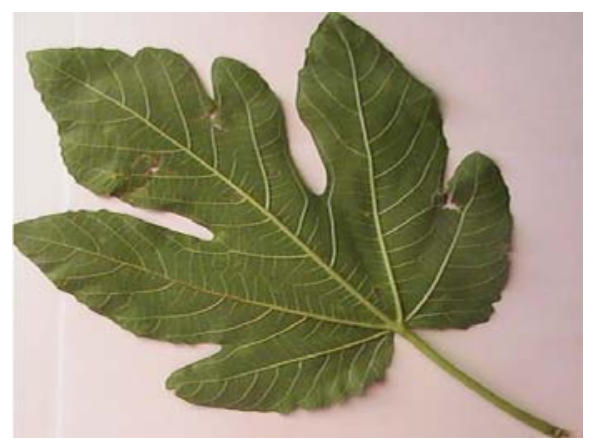

Figure.5 Example of image Fig tree class 3 (Maysony)

Testing set contains 7 pattern for each class, and the Learning set contains 7 patterns for each class (The total is equal to 21 patterns).

The Block diagram of the classification system is shown in figure 2. First of all, the leaf is inputted to the system. Preprocessing does such as converting RGB image to Gray image, filtering, segmentation then the features of the leaf are extracted by using NDBMI, which are used by neural system classifier. (Figures 7, 8 and 9)

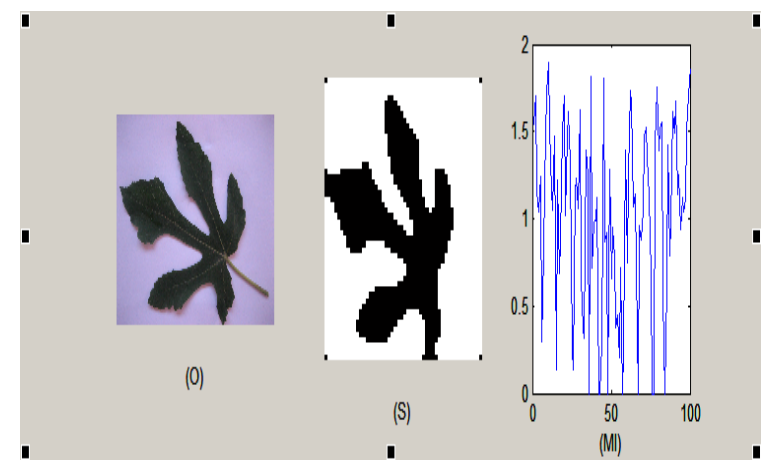

Figure. 6 O: or iginal Image S: Segmented image, M I: mutual information of $\mathrm{S}$ of size $50 \times 50$.

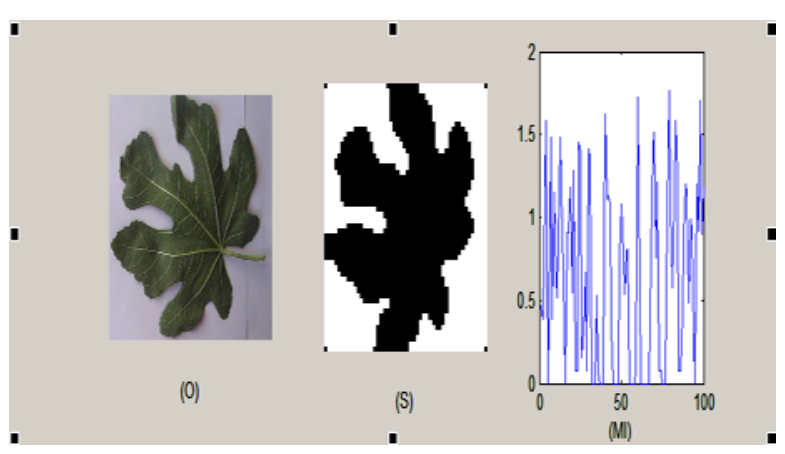

Figure. 7 O: or iginal Image S: Segmented image, MI: mutual information of $S$ of size $50 \times 50$.

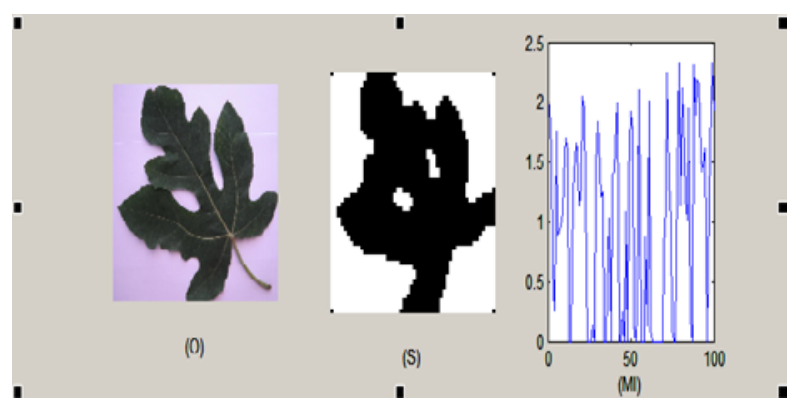

Figure. 8 O: original Image S: Segmented image, MI: mutual information of $\mathrm{S}$ of size $50 \times 50$.

We are appalling the NDBM I descriptor by using the image size $100 \times 100$ and $5 \times 5$ sub images, and then we have a feature vector size 400 . See Fig. 10

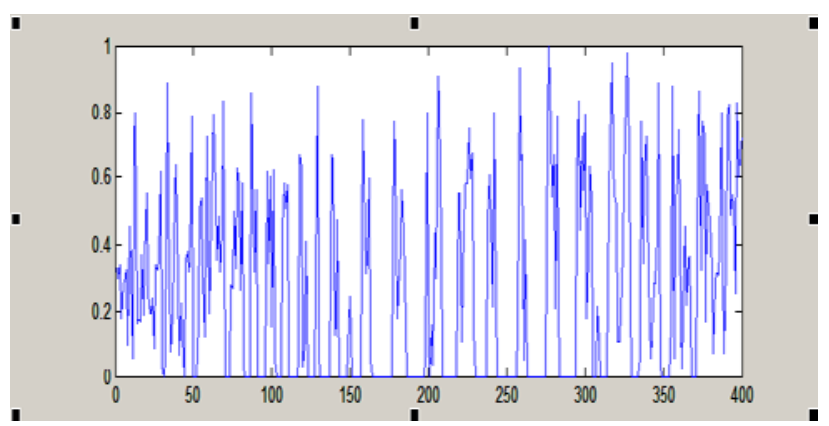

Figure. 9 MI: mutual information of $S$ of size $100 \times 100$.

The experiments were accomplished by using three kinds of FIG trees. Firstly, we are using three pairs of types. Type 1 with type 2, type 2 with type 3 , and type 3 with type1. Secondly, we are using the three types together.

The accuracy of the system is calculated by using the following equation:

$$
\text { Accuracy }=\frac{n_{r}}{n_{t}}
$$

, where $n_{r}$ is the numbers of correct leaves classify and $n_{t}$ is the total number of leaves.

We were obtained accuracy $92.6 \%$ for the classification rate over all the two types classification and accuracy $90 \%$ for the 3 types.

The results are showing in figures 12 and 13 for twoclass classification, where figures 14 and 15 show the classification of the three classes. 


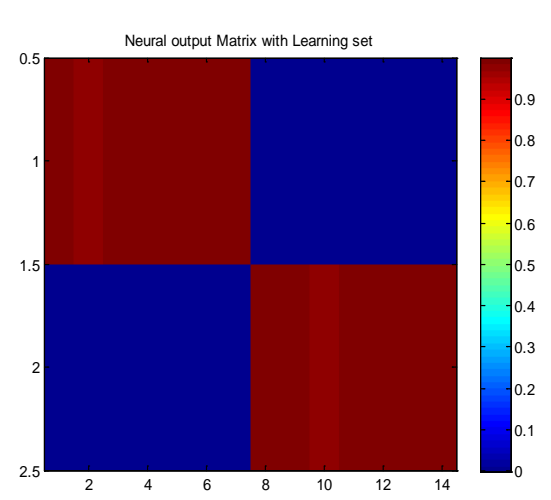

Figure 10-Testing with data Learning (two classes)

Out NN: with 500 iterations

\begin{tabular}{|lllllllllll|}
\hline 0.99 & 0.99 & 0.99 & 0.96 & 0.92 & 0.94 & 0.80 & 0.26 & 0.85 & 0.74 \\
& & & 0.94 & 0.88 & 0.81 & 0.65 & & & \\
\hline 0.29 & 0.06 & 0.97 & 0.99 & 0.36 & 0.90 & 1.00 & 1.00 & 0.98 & 1.00 \\
& & & 0.97 & 0.94 & 1.00 & 0.96 & & & \\
\hline
\end{tabular}

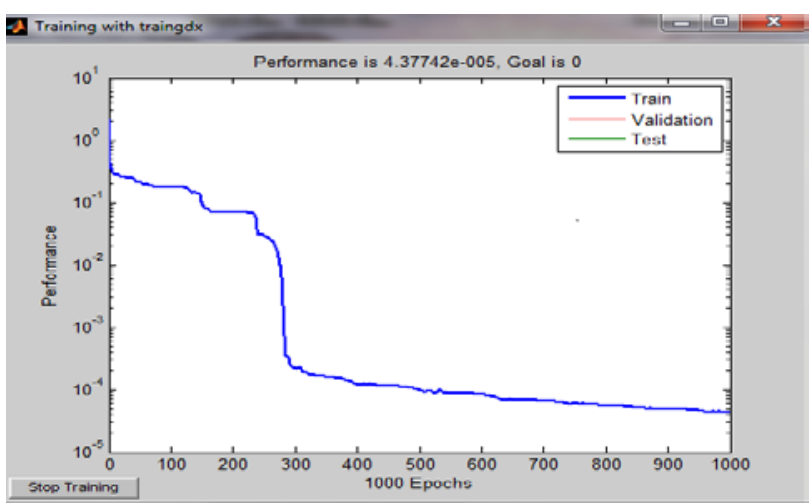

Figure 11: Performance Neural networks out after 1000 iterations.

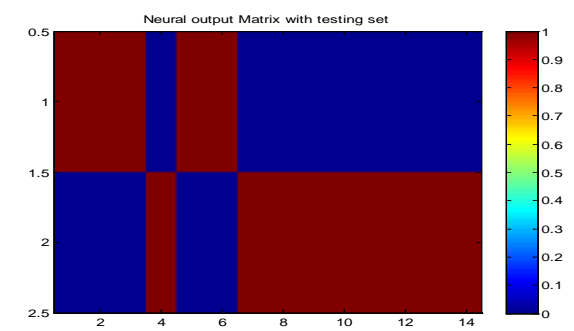

Figure 12- Neural out matrix with Testing set (two classes) 92.8 \% Dir Attia/shaghoury

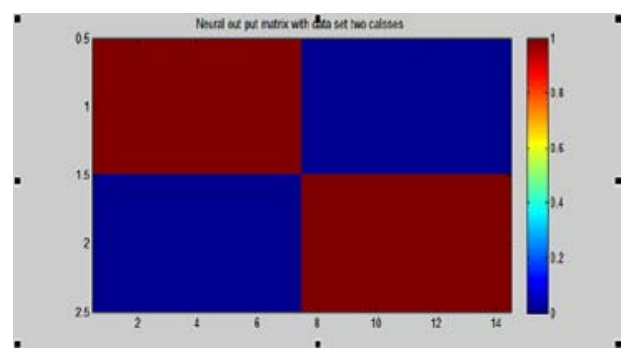

Figure 13- Neur al out matrix with Testing set (two classes) 100\% Dir Attia/shaghoury

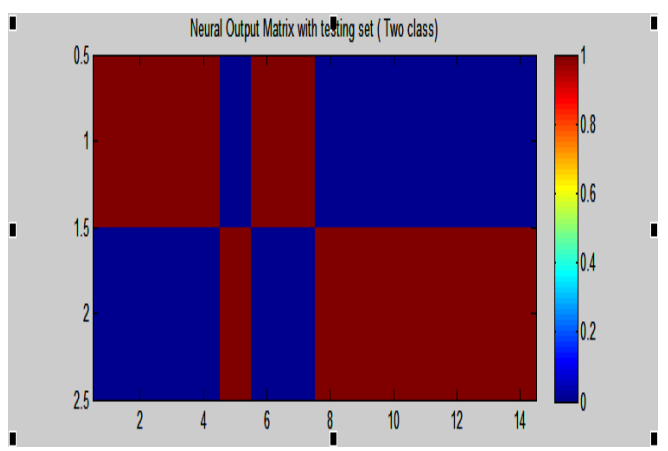

Figure 14- Neural out matrix with Testing set (two classes)

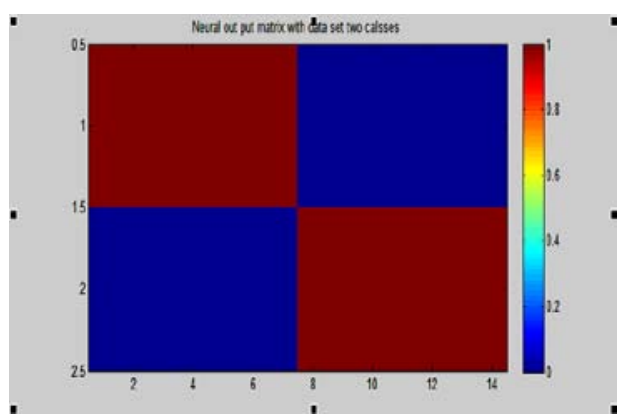

Figure 15- Neur al out matrix with Testing set (two classes) (100\% Maysony /shaghoury)

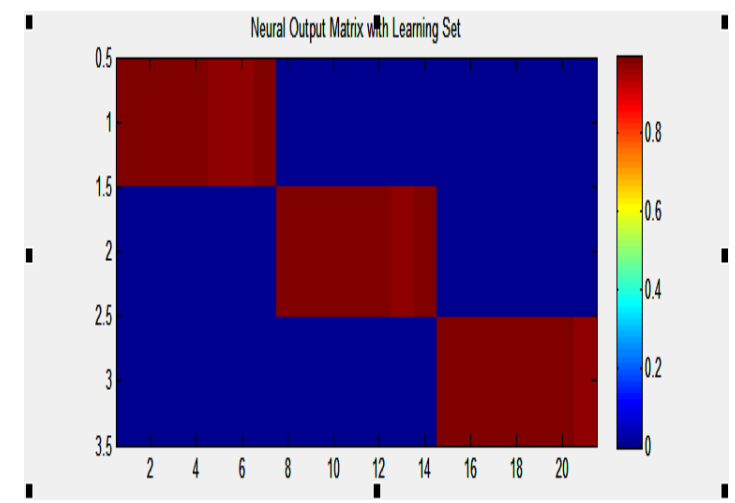

Figure 16- Neur al out matrix with Learning set (3 classes)

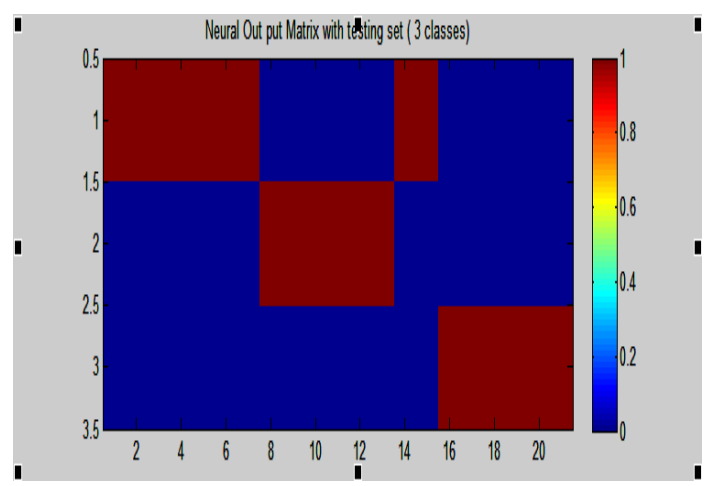

Figure 17- Neural out matrix with testing set (3 classes)

$90.4 \%$

\section{CONCLUSION}

We study and propose new descriptor based on mutual information theory, which used as input to neural 
networks classifier for classification Fig (Ficus Carica L) trees. We use two hidden layer feed forward neural networks. The proposal technique is applied to set of 42 samples ( 3 classes) 14 image for each class. 7 Images of each class are used for the network training and the remaining images are used for generalization and testing. The patterns (images) were described using a new descriptor based in mutual information. The result shows that the recognition rates were $100 \%$ for the training and $92.8 \%$ for generalization. The preliminary results obtained indicated the technical feasibility of the proposed method that will be applied for more than 120 varieties existent in Syria

\section{REFERENCES}

[1] M. Kumar, M. Kamble, S. Pawar, P. Patil and N. Bonde, "Survey on Techniques for Plant Leaf Classification", International Journal of Modern Engineering Research, vol. 1, no. 2, (2011), pp. 538544.

[2]Z. Zulkifli, "Plant Leaf Identification using Moment Invariants \& General Regression Neural Network”, Master Thesis, Universiti Teknologi Malaysia, (2009).

[3] G. Wu, F. S. Bao, E. Y. Xu, Y. X. Wang, Y. F. Chang and Q. L. Xiang, "A Leaf Recognition Algorithm for Plant Classification using Probabilistic Neural Network”, IEEE 7th International Symposium on Signal Processing and Information Technology, (2007).

[4]K. Singh, I. Gupta and S. Gupta, "SVM-BDT PNN and Fourier Moment Technique for Classification of Leaf Shape”, International Journal of Signal Processing, Image Processing and Pattern Recognition, vol. 3, no. 4, (2010), pp. 67-78.

[5] M. Shabanzade, M. Zahedi and S. A. Aghvami, "Combination of Local Descriptors and Global Features for Leaf Classification”, Signal Image Processing: An International Journal, vol. 2, no. 3, (2011), pp. 23-31.

[6] Q. Wu, C. Zhou, \& C. Wang, "Feature Extraction and Automatic Recognition of Plant Leaf Using Artificial Neural Network”, Avances en Ciencias de la Computacion”, pp. 5-12, 2006.

[7] P. Hiremath, \& J. Pujari, "Content based Image Retrieval based on Color, Texture and Shape Features Using Image and Its Complement”, International Journal of Computer Science and Security , vol. 1 (4), pp. 44-50, 2011.

[8] B. Jyothi, Y. M. Latha, \& V. Reddy, "Medical Image Retrieval using Multiple Features", Advances in Computational Sciences and Technology, vol. 3 (3), pp. 387-396, 2010.

[9] S. Theodoridis, \& K. Koutroumbas, “An Introduction Pattern Recognition”, Burlington: Academic Press, 2009.

[10] A. Kulkarni, “Artificial Neural Networks for Image Understanding”, New York: Van Nostrand Reinhold, 1994.
[11] M. Mercimek, K. Gulez, \& T. V. Mumcu, "Real Object Recognition Using Moment Invariants", Sadhana, vol. 30 (6), pp. 765-775, 2005.

[12] D. Zhang, "Image Retrieval Based on Shape", Unpublished Dissertation, Monash University, 2002.

[13] Q. K. Man, C. H. Zheng, X. F. Wang and F. Y. Lin, "Recognition of Plant Leaves using Support Vector", International Conference on Intelligent Computing, (2008), pp. 192-199, Shanghai.

[14] A. Kadir, L.E. Nugroho, A. Susanto and P. I. Santosa, "Leaf Classification using Shape, Color, and Texture Features", International Journal of Computer Trends and Technology, vol. 1, no. 3, (2011), pp. 225-230.

[15] A. Kadir, L.E. Nugroho, A. Susanto and P. I. Santosa, "Neural Network Application on Foliage Plant Identification", International Journal of Computer Applications, vol. 29, no. 9, (2011), pp. $15-22$.

[16] T.M. Cover, J.A. Thomas, Elements of Information Theory, Wiley, New Jersey, 2005.

[17] A.J. Butte, I.S. Kohane, Mutual information relevance networks: functional genomic clustering using pair wise entropy measurements, PSB 5 (2000) 415-426.

[18] G.S. Michaels, D.B. Carr, M. Askenazi, S. Fuhrman, X. Wen, R. Somogyi, Cluster analysis and data visualization of large scale gene expression data, PSB 3 (1998) 42-53.

[19] Herzel H, Grosse I: Measuring correlations in symbols sequences. Physica A 1995, 216:518-542.

[20] Steuer R, Kurths J, Daub CO, Weise J, Selbig J: The mutual information: Detecting and evaluating dependencies between variables.

[21] B. Kröse, P. Van Der Smagt, An introduction to neural networks (8th ed.), 1996, available on http://www.fwi.uva.nl/research/neuro.

[22] C. Bishop, Neural Networks for Pattern Recognition, Clarendon Press, Oxford, UK, 1995.

[23] J.A. Freeman, D.M. Skapura, Neural Networks: Algorithms, Applications and Programming Techniques, Addis on-Wesley, Reading, MA, 1991.

[24] D.E. Rumelheart, G.E. Hinton, R.J. Williams, in: D.E. Rumelheart, J.L. McClelland (Eds.), Parallel Distributed Processing. Explorations in the Microstructure of Cognition, MIT Press, Cambridge, 1986, pp. 318-362.

[25] D.E. Rumelheart, R. Durb in, R. Golden, Y. Chauvin, in: Y. Chauvin, D.E. Rumelheart (Eds.), Backpropagation: Theory, Architectures and Applications, Lawrence Erlbaum, Hillsdale, 1995, pp. 1-34.

[26] D.E. Rumelheart, G.E. Hinton, R.J. Williams, Nature 323 (1986) 533.

[27] Patterson, D. (1996). Artificial Neural Networks. Singapore: Prentice Hall.

[28] Haykin, S. (1994). Neural Networks: A Comprehensive Foundation. New York: Macmillan Publishing. 
[29] G A Azim and M.K. Sousow " Multi Layer Feed Forward Neural Networks For Olive trees Identification" IASTED. Conference on Artificial Intelligence and Application , 11-13 February. (AIA 2008) pp 420-426 Austria.

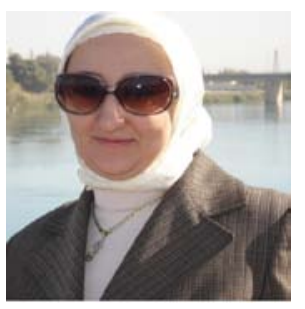

Ghada Kattmah, received her BSc in Agricultural science from Damascus University in 2000 and a Diploma in Horticultural science 2002. She received MSc. degrees in olive resources and genetic improvement from Damascus University, Syria, in 2009. She has worked at General Commission of Agriculture Scientific Research (GCSAR) as researcher assistant, and participated in many cooperative projects with European united, she attended many courses of international olive council on olive production. Her Current conducting research are Essentially about description, evaluation and genetic relationship of olive resources including wild types by using Contemporary techniques, in addition to some researches about pomegranate and fig cultivars. She also works as a trainer in the field of organic system production in cooperative with FAO organization.

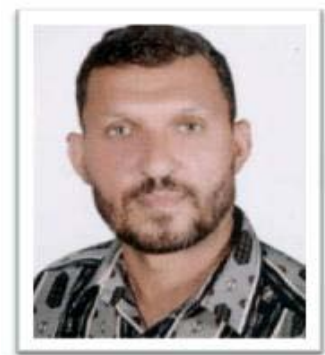

Gamil Abdel Azim, Received his BSc in Mathematics from Cairo University 1979 and a DEPS (Diplome des Etudes Pratiques Superieures) from Poitiers, France. He received MSc. and Ph.D. degrees in Computer Science from Paris Dauphine University, France, in 1988 and 1992, respectively. He worked as Associate Professor in the Department of Computer Sciences, College of Computer and Informatics, Canal Suez University Egypt, and $\mathrm{He}$ worked as an Associate Professor at Computer Science Department, Computer College, Qassim University, Saudi Arabia. His Current research interests include Neural Networks, Combinatorial Optimization, Pattern Recognition, Evolutionary Computation (Genetic algorithms and Genetic Programming), and Bio informatics. He supervised about 30 BSc student projects. Dr. Gamil is Member of IEEE. 